TERRA. Revista de Desarrollo Local e-ISSN: 2386-9968

Número 9 (2021), 60-80

DOI 10.7203/terra.9.21791

IIDL - Instituto Interuniversitario de Desarrollo Local

\title{
¿Es el empleo un factor clave para el asentamiento de población? Una aproximación sociolaboral a la inmigración extranjera en el medio rural vasco (España)
}

\author{
Beatriz Izquierdo Ramírez \\ Profesora Contratado Doctor. Universidad de Burgos \\ bizquierdo@ubu.es \\ https://orcid.org/0000-0002-0137-8182
}

Julia Shershneva

Profesora Adjunta. Universidad del País Vasco (UPV-EHU)

julia.shershneva@ehu.eus

https://orcid.org/0000-0003-3098-9991

Patricia Campelo Martínez

Profesora Agregada. Universidad del País Vasco (UPV-EHU)

patricia.campelo@ehu.es

https://orcid.org/0000-0001-8769-2342

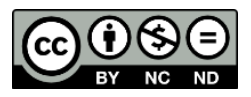

Esta obra se distribuye con la licencia Creative Commons

Reconocimiento-No Comercial-Sin Obra Derivada 4.0 Internacional 


\title{
SECCIÓN ARTÍCULOS
}

\author{
¿Es el empleo un factor clave para el asentamiento de población? Una aproximación sociolaboral a \\ la inmigración extranjera en el medio rural vasco (España)
}

\begin{abstract}
Resumen: Este artículo analiza la presencia de la población extranjera en las zonas rurales vascas con el objetivo de conocer su perfil sociodemográfico y laboral, y valorar la sostenibilidad de su proyecto migratorio, que resulta oscilante según el ciclo económico. Para ello, el estudio examina los principales rasgos de este colectivo e incorpora algunas comparativas con el colectivo de extranjeros residente en las zonas urbanas vascas con el propósito de conocer si existen rasgos diferenciales. Con esta misma finalidad se contrasta la situación laboral de la población rural extranjera, y el rural total. La aproximación metodológica que se utiliza recurre a la explotación estadística confeccionada ad-hoc para este trabajo. Los resultados muestran que la población extrajera presenta un gran potencial para el frenar el despoblamiento y rejuvenecer las zonas rurales en declive. Se trata de un colectivo joven, equilibrado según el género, y con una elevada presencia de la generación soporte. Sin embargo, el análisis también refleja un mercado de trabajo segmentado según el origen de la población que es, además, desigual en cuanto a las condiciones laborales y las actividades que ocupan los trabajadores extranjeros. Una situación que puede desequilibrar la sostenibilidad de su estancia a largo plazo
\end{abstract}

Palabras clave: Población extranjera, medio rural, migración laboral, País Vasco.

\section{Is employment a key factor for demographic settling down? A socio- occupational approach to foreign migration in basque rural areas (Spain)}

Abstract: This paper analyses the presence of international migrant population in the Basque rural areas for the last two decades with the aim of understand their sociodemographic and labour profile, and estimate the sustainability of their migration project which might fluctuate depending on the economic cycle. For that, the research studies the main characteristics of this group and adds some comparisons with the international migrants living in Basque urban areas with the purpose of knowing if there are distinguishing features. Having in mind the same purpose, the labour situation of rural international migrant population is compared with the rural overall ones. The methodological approach focuses on a statistical analysis that has been elaborated ad hoc for this research. The results show that international migrant population presents a huge potential for reversing depopulation and revitalizing the most declining rural areas. They became a group with a significant presence of support generation, being young and gender balanced. However, the analysis also reflects a segmented labour market depending on the origin of population which is, besides, unequal in labour conditions and regarding the working activities foreign population occupy. A situation that might unbalances the sustainability of a longer term stay.

Key words: International migration, rural areas, labour migration, Basque Country.

Recibido: 21 de octubre de 2021

Devuelto para revisión: 15 de diciembre de 2021

Aceptado: 21 de diciembre de 2021

\section{Referencia / Citation:}

Izquierdo, B., Shershneva, J., y Campelo, P. (2021). ¿Es el empleo un factor clave para el asentamiento de población? Una aproximación sociolaboral a la inmigración extranjera en el medio rural vasco (España). TERRA. Revista de Desarrollo Local, (9), 60-80. DOI 10.7203/terra.9.21791 
1. La presencia de migración de origen extranjero oscila en función del ciclo económico.

2. La crisis económica ha modificado el perfil y la actividad laboral de este colectivo

3. Las actividades laborales de la población rural de origen extranjero y autóctono difieren sustancialmente

4. Las ocupaciones de la migración rural de origen extranjero son más precarias $\mathrm{y}$ volátiles.

5. Las condiciones laborales más inestables de este colectivo cuestionan la sostenibilidad de su proyecto migratorio
1. The presence of international migration in Basque rural areas depends on the economic cycle.

2. The economic crisis has modified the working activity of this group.

3. The labour activity profile of international migration and locals in rural areas differs substantially

4. The employment of the rural international migration is more precarious and volatile.

5. The most unstable working conditions of this group enquiries the sustainability of the migration project.
1. La presència de migració d'origen estranger oscil·la en funció del cicle econòmic.

2. La crisi econòmica ha modificat el perfil i l'activitat d'aquest col·lectiu.

3. Les activitats laborals de la població rural d'origen estranger i autòcton difereixen substancialment.

4. Les ocupacions de la migració rural d'origen estranger són més precàries $\mathrm{i}$ volàtils.

5. Les condicions laborals més inestables d'aquest col·lectiu qüestionen la sostenibilitat del projecte migratori. 


\section{INTRODUCCIÓN Y JUSTIFICACIÓN}

La transformación de las zonas rurales en espacios capaces de generar nuevas oportunidades de empleo ha convertido a numerosas poblaciones en foco de atracción de nuevos residentes de origen extranjero ${ }^{1}$, modificando sustancialmente el escenario sociodemográfico precedente. Aunque la presencia de población extranjera en las áreas rurales no es novedosa en España, en los últimos años adquiere una mayor preeminencia no sólo por su incremento en términos cuantitativos, sino por su diseminación en gran parte del territorio, convirtiéndose en uno de los fenómenos de mayor relevancia de las poblaciones rurales (Camarero et al., 2013). Diferentes investigaciones a nivel estatal (Camarero y Sampedro, 2009, 2013, 2019; Escribano, 2012; Solé et al., 2012; Collantes et al., 2014;) subrayan el impacto que genera el asentamiento de este colectivo en el medio rural, aunque también se cuestiona su sostenibilidad a largo plazo.

La mayoría de estas investigaciones sustentan la búsqueda de oportunidades laborales en las zonas rurales como la principal motivación del proyecto migratorio. Coincidiendo con el proceso de llegada de población extranjera a España, las zonas rurales han ido incrementando sus posibilidades de empleo: a la elevada demanda de mano de obra en la agricultura intensiva y agroindustria, se suma la oferta de empleo ligada a la construcción en los años previo a la crisis, así como a la proliferación de empleos auspiciados bajo las políticas de desarrollo rural y vinculadas fundamentalmente al sector servicios. En efecto, el empleo como eje de atracción de nuevos residentes ha favorecido la llegada a las zonas rurales de población extranjera joven en edad de trabajar, lo que impacta directamente sobre el rejuvenecimiento y reequilibrio de género en unas estructuras socioeconómicas que en muchos casos acusaban un imparable declive.

Sin embargo, aunque se asume esta motivación laboral, son más escasos los estudios que incorporan las características del mercado de trabajo de la población extranjera y cuyo análisis supone un factor imprescindible para poder valorar la sostenibilidad de su proyecto migratorio. Hay que tener en cuenta así mismo que la mayoría de investigaciones se elaboran en los años inmediatos a la crisis económica y, aunque anticipan algunos de los posibles efectos sobre la población extranjera en las zonas rurales (Solé et al., 2012; Camarero et al., 2016), no aparecen muchos estudios que aludan con posterioridad a esta temática.

A partir de este planteamiento, el objetivo de la investigación es analizar desde una aproximación cuantitativa el perfil sociodemográfico y laboral de los nuevos residentes extranjeros que se han asentado en las poblaciones rurales vascas de menor tamaño en los últimos veinte años. Se trata de identificar las características de este colectivo según los datos más recientes, y conocer su evolución temporal tomando la crisis económica como referente con la finalidad de identificar los efectos de ésta sobre el proceso migratorio. Pero, además, el trabajo incorpora comparativas de interés: por un lado, se contrastan las dinámicas de la población extranjera en las zonas rurales y urbanas permitiendo identificar los (posibles) elementos diferenciadores del colectivo presente en las zonas rurales. En segundo lugar, se contrastan y analizan los rasgos laborales de la población rural de origen extranjero y la población total, con la idea de conocer su relación con el mercado de trabajo y su situación después de la crisis.

\footnotetext{
${ }^{1}$ Esta investigación ha utilizado como base de datos la población de origen extranjero con el objetivo de incorporar a la población ya nacionalizada. Sin embargo, para una lectura más ágil del texto, a lo largo del mismo aparece únicamente el nombre de población extranjera.
} 
Todas estas cuestiones abren sin duda amplios interrogantes acerca de las características de los migrantes extranjeros asentados en estas zonas. Conocer dónde se ubican, cuáles son sus rasgos sociodemográficos y las principales zonas de origen desde una perspectiva longitudinal (y comparada con el residente en zonas urbanas) va a favorecer la posibilidad de abordar planteamientos de gran interés. Además, desde la perspectiva del mercado de trabajo, es importante conocer la estabilidad de los empleos que ocupa la población extranjera, sus ámbitos de empleo y condiciones laborales. Esto es, se trata de indagar acerca de las actividades que realizan, si se trata de un posible reemplazamiento de mano de obra autóctona por mano de obra extranjera o conocer el papel de las mujeres trabajadores en este escenario entre otras cuestiones principales.

Para finalizar, los apartados siguientes desarrollan esta propuesta e incorporan los principales hallazgos obtenidos a partir de la explotación de datos estadísticos confeccionados varios de ellos ad hoc para este estudio. La revisión teórica de las principales aportaciones sobre este tema sirve de marco de estudio para definir las orientaciones que guían la investigación que justifica, así mismo, la idoneidad de la ruralidad vasca como escenario de acogida de la población extranjera

\section{BASES TEÓRICO-CONCEPTUALES. LA POBLACIÓN EXTRANJERA EN LAS ZONAS RURALES COMO OBJETO DE ESTUDIO}

La llegada de población extranjera a las zonas rurales se ha planteado en la mayoría de estudios como una oportunidad para revertir o, al menos, mitigar el proceso de despoblamiento y envejecimiento de las poblaciones rurales en España (Marcilla et al., 2011; Collantes et al., 2014). No obstante, y a pesar de las expectativas generadas, investigaciones más recientes (Camarero y Sampedro, 2019) reconocen que la distribución de este colectivo en las zonas rurales ha sido desigual, teniendo un mayor impacto en las poblaciones mejor comunicadas. Otros estudios a nivel europeo comparten esta misma percepción (Rye y Sletteback, 2020) y detectan que las personas inmigrantes se localizan fundamentalmente en las comunidades más vitales.

Más allá de su potencial impacto, la mayoría de estas investigaciones incluidas las que incorpora en el apartado anterior, reflejan la motivación laboral como motor principal para el asentamiento en estas poblaciones. Una realidad que va a favorecer que su distribución e impacto territorial vaya a depender en gran medida de las oportunidades laborales que ofrezcan esto lugares. En los últimos años, las zonas rurales han ido generando posibilidades de empleo en los sectores agrícola y servicios; dentro del ámbito agrario, destaca la progresiva demanda de mano de obra en la agricultura intensiva y agroindustria. Diferentes investigaciones se han centrado en estos sectores reflejando en gran medida el incremento de empleos flexible y poco cualificado en estos sectores en los que prevalecen unas condiciones sociolaborales precarias (Torres y Gadea, 2010; Danson y Jentsch, 2012; Gualda, 2012; Gadea et al., 2015; Gadea et al, 2016). En el contexto europeo, algunos estudios muestran una situación similar, y destacan cómo el reclutamiento de trabajadores inmigrantes especialmente vulnerables para asegurar una mano de obra barata, dócil, y flexible ha sido clave en el progresivo incremento de la industrialización agraria (Rye y Scott, 2020).

Pero además de estas actividades, en los últimos años el sector servicios también ha generado oportunidades de empleo en los territorios rurales, favoreciendo en gran medida la incorporación de mujeres extranjeras en ámbitos muy feminizados como los cuidados 
(Solé et al., 2012; Soroellas et al., 2013; Vercher et al., 2019, 2020). En efecto, en este nuevo escenario laboral estos estudios reflejan el positivo impacto que genera la "refeminización" de las zonas rurales, aunque matizan la precarización de los empleos que ocupan muchas de ellas. La agudización del envejecimiento rural y la puesta en marcha de políticas sociales asociadas a la mejora y cuidado de la población mayor en su lugar de residencia ha favorecido este tipo de trabajo, convirtiéndose en algunos casos la entrada al mercado laboral rural de muchas mujeres de origen extranjero. Las autoras no eluden la necesidad de conocer el alcance de la economía sumergida dentro de estas actividades cuya realidad se intensifica en las zonas rurales. No obstante, más allá de esta esfera, la población extranjera se ha incorporado también a tareas ligadas a los sectores turísticos y hosteleros, que, implementadas bajo el paraguas de las políticas de desarrollo rural, ofrecen nuevas oportunidades laborales también para la población extranjera.

Además, muchos de estos estudios se realizan en los años inmediatos a la crisis económica por lo que algunos (son más bien escasos) han abordado también los posibles efectos de la crisis en el mantenimiento de la población extranjera. Mientras que algunos estudios sugieren que la crisis ha provocado un deterioro en las condiciones laborales de estos trabajadores (Torres y Gadea, 2010), otros se plantean los efectos que pueda generar sobre las dinámicas migratorias cuestionando la capacidad de resistencia y adaptación de este colectivo ante un contexto tan desfavorable (Solé et al., 2012; Soroellas et al., 2013).

Para finalizar, junto a la motivación más instrumental de búsqueda de empleo otros estudios plantean interesantes perspectivas de análisis que reflejan cómo otros factores como la composición familiar del colectivo de personas de origen extranjero pueden resultar así mismo determinantes en su asentamiento en las zonas rurales. Para Camarero y Sampedro (2016), la centralidad de la estrategia familiar ayudaría a explicar, por ejemplo, la permanencia de unos colectivos "más resilientes" con expectativas de permanencia más a largo plazo frente a otros mucho más "volátiles" y dependientes de la coyuntura económica.

A partir de estas aportaciones, la investigación realiza una aproximación a la población extranjera que reside en las poblaciones rurales vascas más pequeñas. Un planteamiento cuyos objetivos y metodología se detallan en el apartado siguiente en el que se incorpora también una breve aproximación a la ruralidad vasca destacando aquellos rasgos que ayuden a comprender la presencia (oscilante) de este colectivo en estas zonas.

\section{OBJETIVOS, FUENTES DE INFORMACIÓN Y ÁREA DE ESTUDIO}

El objetivo principal de este trabajo es analizar los rasgos sociodemográficos y laborales de las personas de origen extranjero que residen en las poblaciones rurales vascas ${ }^{2}$. Conocer las características de estos nuevos residentes arroja información de gran interés para poder estimar el alcance y sostenibilidad del asentamiento de estas personas en las zonas rurales. Su edad, balance de género y situación en relación al mercado de trabajo son, entre otras, variables que tienen un impacto significativo y ayudan a promover un equilibrio territorial amenazado en algunos casos por el envejecimiento o despoblación.

\footnotetext{
${ }^{2}$ Siguiendo los estudios centrados en la ruralidad vasca de Camarero y Oliva (1999), se ha acotado numéricamente la consideración de población "rural", a aquellos municipios de igual o menos de 2.000 habitantes teniendo en cuenta exclusivamente el acotamiento numérico.
} 
La motivación laboral como factor principal desencadenante del proyecto migratorio se plantea como primera hipótesis, ya que la obtención de una fuente de ingreso a través del empleo avala en gran medida la viabilidad de este proyecto. En este contexto, la evolución de los saldos migratorios en los años previos y posteriores a la crisis de 2008 va a permitir avalar o refutar esta hipótesis principal.

Otra de las hipótesis que se plantean guarda relación con el perfil sociodemográfico diferencial de los colectivos que se asientan en las zonas rurales y urbanas. Es decir, se trata de conocer si las zonas rurales reciben a un perfil que difiere del que llega a las zonas urbanas. Además, es muy interesante observar el espacio temporal en el que se producen estas llegadas; esto es, si la llegada a las zonas rurales y urbanas se produce de forma simultánea o si, por el contrario, la población extranjera ha llegado más tarde a las zonas rurales.

Por último, el estudio indaga en torno a los principales rasgos del mercado de trabajo y aporta resultados novedosos que aparecen ausentes en muchas de las investigaciones previas debido a la dificultad de disponer datos de empleo en las poblaciones más pequeñas. Se parte de la hipótesis de un mercado de trabajo segmentado en función del origen de los trabajadores, y del género; para ello, se recopilan datos de ocupación y se establece de una comparativa entre la población de origen extranjero y la población total. Además, se analizan las ocupaciones laborales en función de los diferentes orígenes de la población extranjera.

\subsection{Metodología y fuentes de información}

La aproximación metodológica que se ha utilizado se centra en la explotación y análisis de las principales fuentes estadísticas de población y de empleo, utilizando diferentes fuentes secundarias. Por un lado, para la caracterización demográfica se han analizado los Padrones de Población continuos elaborados por el Instituto Nacional de Estadística (INE) de los años 2019, 2016, 2011 y 2006, así como datos procedentes del Instituto Vasco de Estadística (EUSTAT). Se ha optado por explotar la información de estos años para poder obtener la imagen de la inmigración en diferentes etapas: la etapa inicial previa a la llegada masiva (2001), la etapa de pre-crisis (2006), los efectos de la crisis económica (2011), el descenso de los flujos migratorios (2016) y, finalmente, se han añadido los últimos datos de 2019 para conocer la evolución y ofrecer datos que reflejen la situación lo más actualizada posible.

En relación a la caracterización del mercado de trabajo, una de las principales dificultades es el acceso a datos estadísticos referida a los municipios más pequeños. En este estudio, se ha procedido a la petición de datos $a d$ hoc al EUSTAT ${ }^{3}$, entidad que elabora sus propias estadísticas laborales para todos los municipios vascos nutriéndose de la base de datos que recopila y proporciona el Servicio Vasco de Empleo (LANBIDE). Es importante indicar que para confeccionar las Tasas de actividad (y ocupación y paro) se han utilizado las Tasas globales; esto es, teniendo en cuenta como unidad de análisis a la población y no al municipio, evitando problemas de sobrerrepresentación estadística. Además, toda

\footnotetext{
${ }^{3}$ Es importante indicar que la clasificación que utiliza el EUSTAT para la variable de mercado de trabajo contiene categorías no excluyentes, es decir, la suma de todas las categorías no es el total de la población. Así, por ejemplo, las personas activas para un determinado cruce es la suma de parados y ocupados en dicho cruce, así mismo los parados incluyen a los que buscan primer empleo y los que han trabajado.
} 
la información facilitada se ha explotado a partir de tres variables fundamentales: género, edad y país de procedencia o, en su caso, continente.

\subsection{La ruralidad vasca como escenario de asentamiento de la población extranjera}

A diferencia de otros territorios, las zonas rurales del País Vasco se han ido transformando en los últimos años en una ruralidad heterogénea y muy cambiante; un área (o más bien áreas) en las que zonas caracterizadas por un gran dinamismo demográfico conviven con otras que presentan signos evidentes de declive (más habituales en el territorio alavés excluyendo las zonas limítrofes a su capital). En el primer caso, la transformación de estas poblaciones se ha desarrollado fundamentalmente desde finales de la década de los noventa como consecuencia del trasvase de población desde las zonas urbanas a las rurales (Camarero y Oliva, 1999; Ainz Ibarrondo, 2001; Alberdi, 2016; Urrestarazu y Galdos, 2019). En efecto, este tipo de ruralidad se inserta de lleno en el fenómeno de la contra-urbanización que se desarrolla en el País Vasco en ese periodo, aunque quizás con un impacto menor o menos estudiado que en el caso de otras regiones cercanas como Navarra (Rivera, 2009). No obstante, esta metamorfosis no va a afectar a todo el territorio rural y se localiza fundamentalmente en las zonas que cuentan con un mayor atractivo y están mejor comunicadas: las poblaciones costeras de Bizkaia y Gipuzkoa, y, en el caso alavés, en torno al cinturón alrededor de su capital, Vitoria-Gasteiz. El impacto sociodemográfico sobre estas poblaciones ha sido muy significativo al asentarse nuevos residentes mucho más jóvenes y con una elevada presencia de mujeres. Sin embargo, los efectos de este colectivo sobre su estructura laboral han sido limitados ya que muchos de ellos han trabajado fuera del municipio. En efecto, un rasgo característico de esta nueva ruralidad es la intensa movilidad que se produce entre los municipios rurales y las zonas urbanas o semi-urbanas.

Frente a esta realidad, en una investigación reciente centrada en la ruralidad vasca, Urrestarazu y Galdos (2019) se hacen eco de la significativa transformación laboral de las poblaciones rurales. El estudio subraya la capacidad de generar empleo de estos territorios en los que se observa dos fenómenos que transcurren en paralelo: la paulatina terciarización de la economía rural, y la progresiva desagrarización de su población. Los autores subrayan cómo a pesar de la ralentización en la creación de empleo respecto a las zonas urbanas, las poblaciones rurales presentan un ritmo de crecimiento mucho más vivo, por lo que las diferencias se van reduciendo. A diferencia de otras zonas rurales, junto al sector servicios, esta misma investigación destaca el relevante peso de la industria entre la población rural, más perceptible en zonas del interior de Gipuzkoa; un elemento sin duda diferenciador respecto a otros territorios rurales.

En definitiva, las áreas rurales vascas se convierten en este momento en un escenario muy favorable para el asentamiento de población extranjera. Un estudio preliminar elaborado para este estudio refleja con nitidez la distribución de este colectivo en función de la actividad laboral. Así, se detectan tres focos principales en los que se concentran los municipios con una presencia de población extranjera superior al 15\%: la zona de la Rioja alavesa ligada a la industria vitivinícola, las poblaciones del norte alavés en las que predomina la agricultura intensiva, y la zona del interior de Gipuzkoa cercana a los espacios más industrializados. Una realidad que confirmaría la hipótesis de la motivación laboral como factor de asentamiento en las zonas rurales. 


\section{4. ÁREA DE ESTUDIO}

Este apartado expone los principales hallazgos obtenidos en la explotación estadística. Para ello, se realiza un análisis sociodemográfico a partir de los últimos datos disponibles (2019), y se incorpora un análisis longitudinal con el objetivo de conocer los posibles efectos de la crisis económica en el proceso migratorio. La situación de la población en relación al mercado de trabajo se analiza en el siguiente sub-apartado y para ello se establece una comparativa en torno a los principales marcadores laborales de la población extranjera y la población rural total ${ }^{4}$.

\subsection{Principales rasgos sociodemográficos desde una perspectiva comparada}

Uno de los primeros resultados que se extraen es la progresiva presencia de las personas extranjeras, incrementando su representatividad del 1,6\% en 2001 al 7,4\% en 2019. Aunque entre 2006 y 2011 es el periodo en el que se produce un mayor asentamiento de población $(3,6 \%$ al $6,2 \%)$ coincidiendo con la época de bonanza económica, la mayor representatividad se produce en los últimos años y, más en concreto, en el último año que se utiliza como referencia en este estudio (2019).

Figura 1. Distribución de la población extranjera y autóctona en las zonas rurales según género y edad (2019)

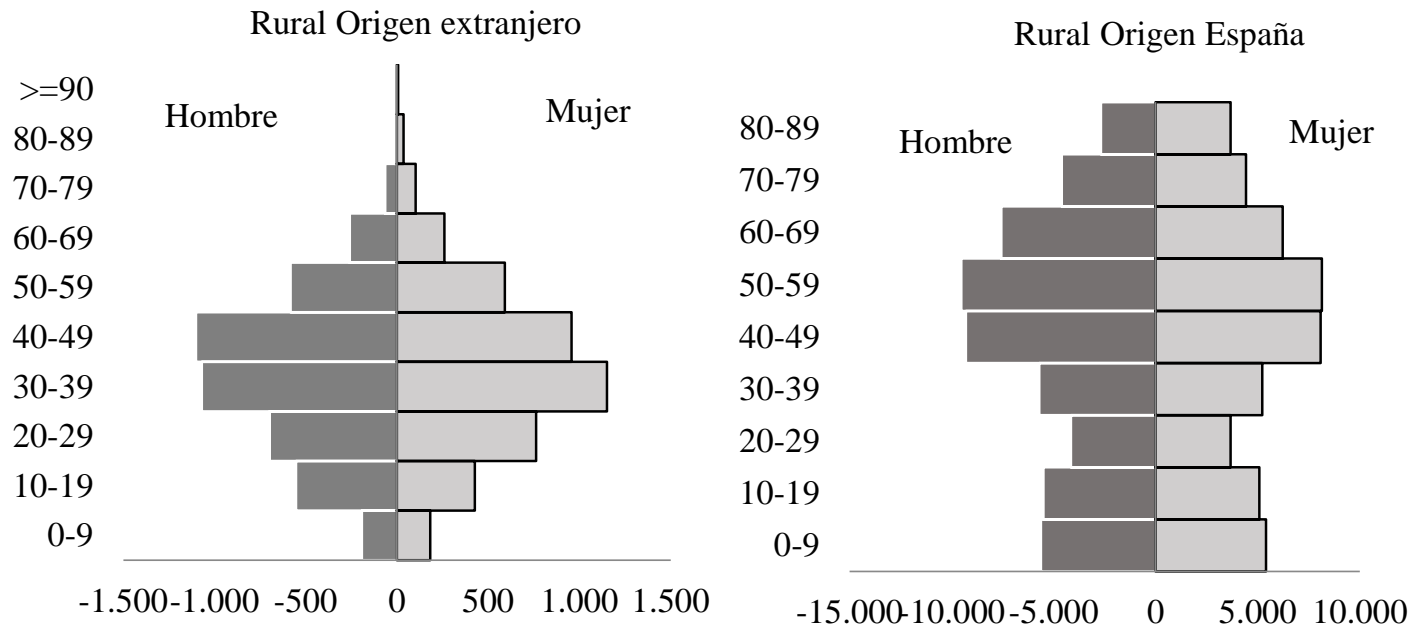

Fuente: elaboración propia a partir de EUSTAT.

Precisamente, el análisis sociodemográfico que se realiza sobre este año último año refleja una composición poblacional heterogénea y muy dinámica. Por un lado, se observa una estructura de género equilibrada aunque muy ligada al origen ${ }^{5}$, lo que puede favorecer

\footnotetext{
${ }^{4}$ No ha sido posible recoger y analizar los datos acerca del mercado de trabajo de la población rural autóctona (excluyendo a la población de origen extranjero o extranjera) al no estar disponible en el EUSTAT, por lo que se ha optado por establecer una comparativa con la población rural total.

${ }^{5}$ En este caso, las mujeres latinoamericanas representan el $62.7 \%$ del total, mientras que entre la población magrebí los hombres alcanzan el $60.1 \%$. Otras procedencias presentan un mayor equilibrio, como la población de origen europeo.
} 
que se modifique en función de la prevalencia de determinados colectivos (más o menos feminizados) en estas zonas. Sin embargo, la edad sí aparece como una variable constante en todos los casos reflejando un perfil muy joven en el que el $82 \%$ del total se encuentra en edad de trabajar. En este caso, además, se ha considerado interesante establecer una comparativa entre la población de origen extranjero y la autóctona con la finalidad de conocer el posible impacto sociodemográfico de los nuevos residentes (Figura 1). En el caso de la población extranjera, es significativo el peso más reducido de los menores de 19 años. Una situación que podría explicarse tanto por las migraciones en solitario como por la adquisición de la nacionalidad española de algunos de los hijos nacidos ya en España.

Las diferencias entre ambos colectivos son evidentes sobre todo en el peso de la generación soporte; es decir, la representación de aquellas personas que por su posición vital constituyen la generación más activa (30 a 49 años) desde la perspectiva de las actividades productivas y reproductivas (Camarero, 2009). Esta tasa es del 47,5\% para las personas de origen extranjero manteniéndose estable desde el inicio, mientras que se reduce al $26,7 \%$ para la población autóctona reduciéndose de forma progresiva.

Junto a la caracterización demográfica, se ha querido indagar también acerca del efecto de la crisis en el proceso migratorio. Para ello, se ha establecido una comparativa entre los saldos migratorios de las personas extranjeras en las zonas rurales y urbanas (Figura 2). Como se observa, desde 2007 hasta 2012 se observa que el ritmo del crecimiento es similar en los dos contextos: tanto en el ámbito rural como en el no rural el crecimiento de la población se detiene a partir de 2009 coincidiendo con la crisis económica. En el año 2013, en cambio, se registra por primera vez un saldo negativo, siendo este el más elevado en 2015 en las zonas rurales (-512), mientras que en los municipios no rurales sucede en 2014 (-1.681). Unos datos que dejar entrever que la población de origen extranjero empezó a abandonar las zonas rurales más tarde que las zonas no rurales.

Figura 2. Saldo migratorio interanual en números absolutos de las personas extranjeras residentes en las zonas rurales y no rurales (2007-2019)

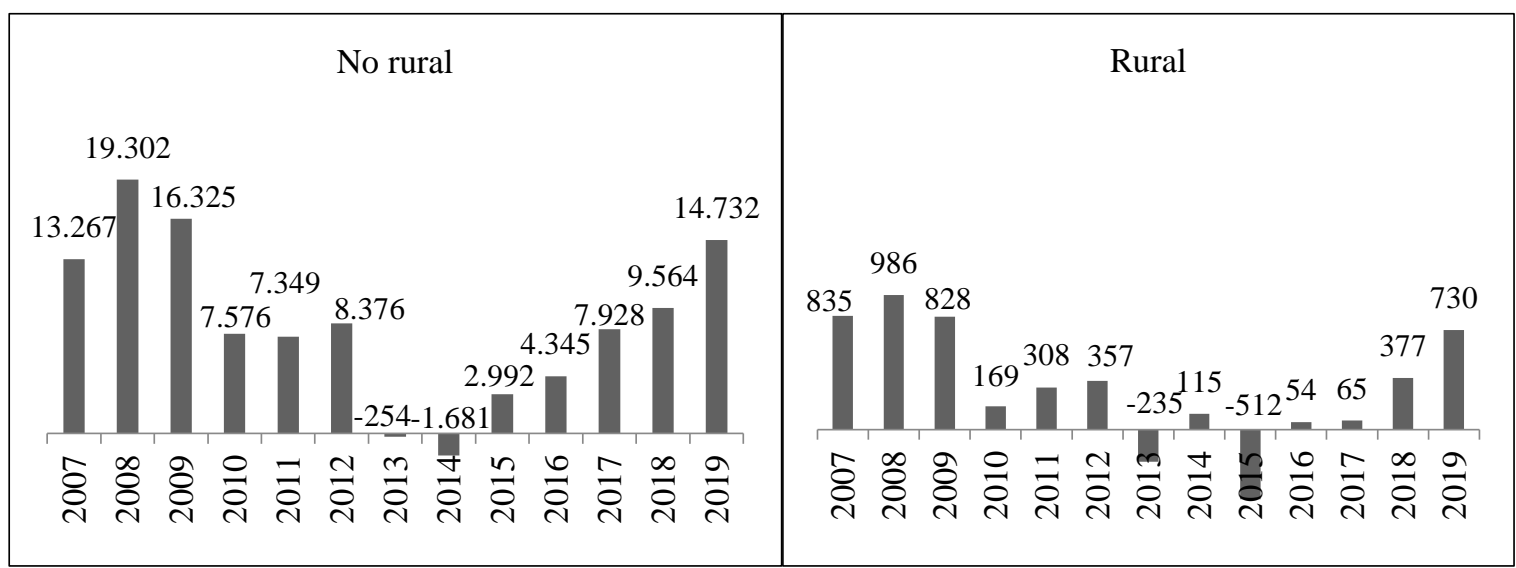

Fuente: elaboración propia a partir de EUSTAT.

Finalmente, en la etapa de la recuperación económica, que en el País Vasco comienza en el año 2015, los flujos migratorios post-crisis se dirigen sobre todo a las zonas no rurales, puesto que es allí donde el ritmo de recuperación es más elevado, mientras que en los municipios rurales no se da hasta 2018, ascendiendo a los niveles de 2012 (337 personas más que el año anterior). 
Este análisis incorpora también la procedencia de la población extranjera a partir de las principales áreas geográficas, para lo que se establece de nuevo una comparativa entre las zonas rurales y urbanas. Aunque las personas de origen latinoamericano son el colectivo más significativo en todo el territorio vasco, se detectan algunas diferencias. En las zonas rurales, la distribución de la población latinoamericana $(37,4 \%)$, no difiere en gran medida del de la población de origen europeo $(30,1 \%)$ y africano $(27,9 \%)$ cuya procedencia es mayoritariamente magrebí. Sin embargo, en las zonas urbanas se observa una prevalencia clara de la población de origen latinoamericano $(50 \%)$ seguida a mucha distancia de la europea y africana, cuya representación es del $21 \%$ en cada caso.

Un análisis longitudinal más detallado (2006-2019) de estos colectivos permite conocer cómo esta distribución ha permanecido prácticamente invariable en el caso de la población latinoamericana, aunque ha evolucionado de forma dispar en el caso de las poblaciones europeas y africanas disminuyendo el peso relativo de los primeros y aumentando la presencia de la población (sobre todo magrebí) en los últimos años. Una representación que va a ser clave para comprender las dinámicas y caracterización del mercado de trabajo del colectivo migrante a lo largo de este periodo.

\subsection{El mercado de trabajo como eje de desigualdad}

El análisis de los principales indicadores del mercado de trabajo entre la población extranjera y la población total reflejan una tasa de actividad global muy similar entre ambos colectivos (56,2 y 58,7), aunque el desglose por género refleja cómo es precisamente esta variable y no el origen la que explica la desigual vinculación de las mujeres al mercado de trabajo, sobre todo extranjeras (Tabla 1$)^{6}$. Aun así, la variable origen sí es clave para analizar la diferencia tan elevada que se observa en la tasa de paro, y en el que las mujeres extranjeras son, sin duda, el colectivo más perjudicado con una tasa cercana al $30 \%$. A priori, estos datos podrían reflejar una presencia mucho más reducida de las mujeres extranjeras en el mercado de trabajo rural, aunque como apuntan estudios previos se desconoce el alcance real de su presencia en la economía sumergida.

En cuanto a la población ocupada, los datos analizados del EUSTAT reflejan un perfil de trabajador cuya representatividad masculina es más elevada $(58,5 \%)$, y una edad media muy joven en el que el $70 \%$ tienen menos de 44 años. Si se distribuye esta población según su origen se percibe una ligera sobre-representación de los hombres ocupados de origen europeo en relación a su representatividad total, y una menor presencia de las trabajadoras de origen latinoamericano y magrebí.

Tabla 1. Datos globales sobre el mercado de trabajo y en función del género y origen (2019)

\begin{tabular}{|c|c|c|c|c|}
\hline & \multicolumn{2}{|c|}{ Población extranjera } & \multicolumn{2}{c|}{ Población Total } \\
\hline & Hombres & Mujeres & Hombres & Mujeres \\
\hline Tasa Global de actividad & 60,4 & 51,9 & 62,1 & 55,1 \\
\hline Tasa Global de paro & 21,6 & 29,8 & 6,9 & 9,7 \\
\hline
\end{tabular}

Fuente: elaboración propia a partir de EUSTAT.

\footnotetext{
${ }^{6}$ Esta diferencia en la tasa de actividad es similar a la media que refleja la Encuesta de Población Activa (EPA) para la población española en 2019 (INE, 2019).
} 
Continuando con las actividades laborales, se ha considerado de interés establecer una comparativa entre aquellas que desarrollan la población extrajera, y la población total integrando además la perspectiva de género (Tabla 2). Los datos muestran la preeminencia de determinadas ocupaciones en ambos grupos; actividades económicas que se desarrollan en ámbitos distintos y, además, desiguales. Por un lado, los hombres extranjeros se han incorporado a tres actividades principales: la agricultura, la industria y la construcción, empleos que aglutinan prácticamente el $60 \%$ del total de las ocupaciones; es significativa y a valorar como un elemento muy positivo la proporción de aquellos que se dedican a la industria teniendo en cuenta las óptimas condiciones laborales de este sector. Le siguen a bastante distancia las vinculadas al comercio y hostelería. Las mujeres extranjeras, por el contrario, se concentra básicamente en el sector servicios $(89,2 \%)$ y en dos ámbitos principales: la hostelería y, sobre todo, el empleo doméstico. Ocupaciones en ambos casos con una temporalidad y unas condiciones laborales, a priori, mucho peores.

Tabla 2. Distribución por principales actividades económicas de la población ocupada extranjera y población total diferenciado según sexo (\%) (2019)

\begin{tabular}{|c|c|c|c|c|}
\hline & \multicolumn{2}{|c|}{ Población extranjera } & \multicolumn{2}{c|}{ Población total } \\
\hline & Hombres & Mujeres & Hombres & Mujeres \\
\hline Agricultura, ganadería, caza, silvicultura & 16,9 & 3,7 & 7,0 & 3,6 \\
\hline Industria manufacturera & 21,7 & 6,4 & 35,0 & 11,3 \\
\hline Construcción & 19,7 & 0,7 & 8,9 & 1,3 \\
\hline Comercio y reparación & 9,9 & 10,1 & 9,7 & 12,3 \\
\hline Hostelería & 9,0 & 20,1 & 4,1 & 9,8 \\
\hline $\begin{array}{c}\text { Alquiler, inmobiliarias y servicios a } \\
\text { empresas }\end{array}$ & 8,7 & 10,6 & 8,5 & 10,8 \\
\hline Educación & 2,3 & 5,1 & 4,7 & 15,5 \\
\hline $\begin{array}{c}\text { Actividades sanitarias y veterinarias; } \\
\text { servicios sociales }\end{array}$ & 1,6 & 7,9 & 2,9 & 14,05 \\
\hline $\begin{array}{c}\text { Otras actividades sociales y servicios } \\
\text { personales }\end{array}$ & 1,5 & 4,6 & 2,8 & 5,2 \\
\hline Hogares que emplean personal doméstico & 1,8 & 26,8 & 0,2 & 3,8 \\
\hline Otras actividades económicas & 6,9 & 3,7 & 6,9 & 12,5 \\
\hline Total & 100 & 100 & 100 & 100 \\
\hline
\end{tabular}

Fuente: elaboración propia a partir de EUSTAT.

La distribución laboral de la población extranjera contrasta con las actividades de la población total, con un rango de ocupaciones más elevado y, a priori, con mejores condiciones de empleo. Por un lado, las actividades que realizan los hombres presentan una proporción de ocupados en la industria muy significativa lo que revela, como se adelantaba en páginas anteriores, el carácter diferencial de la ruralidad vasca frente a otras zonas. Le sigue a bastante distancia otras actividades como el comercio o la construcción. Destaca además la reducida proporción de aquellos que se dedican a la agricultura, con una representación frente a la de los hombres extranjeros de 10 puntos menos. En el caso de las mujeres, las actividades se concentran también en el sector servicios, pero a diferencia de las mujeres extranjeras, su distribución resulta mucho más equilibrada 
ocupando un rango de actividades más elevado y con mayor cualificación (ocupaciones sanitarias, educativas, comercio etc.).

En el caso de la población extranjera se han considerado interesante realizar un análisis evolutivo de las principales actividades económicas (2001-2019) con el objetivo de detectar si se ha producido una transformación laboral en los últimos años y, asimismo, conocer si la crisis ha supuesto un eje diferenciador en estas actividades. Por un lado, la revisión de datos refleja el incremento de actividades como la agricultura, el comercio y la hostelería; ocupaciones que se habrían incrementado en unos cuatro-seis puntos en los últimos 14 años. Sin embargo, son las actividades de persona empleada de hogar las que han sufrido un mayor repunte (en 10 puntos), sobre todo a partir del año 2016; un proceso directamente conectado con el progresivo proceso de envejecimiento de la población rural. Frente a estas, la construcción ha perdido más de 10 puntos en este periodo, aunque como se ha podido observar, continúa teniendo un peso importante. Así mismo, la industria manufacturera es otro de los sectores que ha ido perdiendo un cierto peso, sobre todo en los últimos tres años, aunque la proporción de hombres extranjeros ocupada en este sector es aun significativa.

Por último, y tomando los datos de 2019, se ha elaborado un análisis más detallado que vincula los principales sectores económicos y la población extranjera según su procedencia principal (por continentes a excepción población magrebí) ${ }^{7}$. No se ha podido desglosar las actividades más allá del sector al no estar disponibles en el EUSTAT según el origen de la población. En este caso, la etno-estratificación que aparece con claridad en otros estudios (Gadea, 2015, 2016), se detecta únicamente en el caso la agricultura, actividad cuya representatividad es mayor entre la población magrebí y, en especial, entre los hombres (Tabla 3). Entre las mujeres magrebíes la tasa de incorporación a la agricultura también es mayor que en el resto, aunque poco significativa sobre el total, con una clara prevalencia del sector servicios $(83 \%)$.

Tabla 3. Distribución de la actividad laboral por sectores de la población extranjera ocupada según procedencia y género (\%) (2019)

\begin{tabular}{|c|c|c|c|c|c|c|}
\hline & \multicolumn{2}{|c|}{ Pobl. origen magrebí } & \multicolumn{2}{c|}{ Pob. origen latinoamericano } & \multicolumn{2}{c|}{ Pob. origen europeo } \\
\hline & Hombres & Mujeres & Hombres & Mujeres & Hombres & Mujeres \\
\hline Agricultura & 26,1 & 7 & 14,2 & 2,1 & 18,0 & 5,3 \\
\hline Construcción & 12,1 & 2 & 10,3 & 0,5 & 28,0 & 0,5 \\
\hline Servicios & 40,5 & 83 & 55,7 & 93,9 & 34,6 & 86,5 \\
\hline Industria & 22,2 & 8 & 19,6 & 3,5 & 19,4 & 7,7 \\
\hline Total & 100 & 100 & 100 & 100 & 100 & 100 \\
\hline
\end{tabular}

Fuente: elaboración propia a partir datos EUSTAT.

En relación a la población latinoamericana, la actividad laboral de los hombres es más equilibrada que en el caso de las mujeres. Mientras que ellas se concentran de forma masiva en el sector servicios (91\%), los hombres se distribuyen entre otros sectores, aunque prevalece así mismo este sector. En tercer lugar, las mujeres ocupadas de origen europeo presentan una distribución muy similar a las mujeres magrebíes, aunque los

\footnotetext{
${ }^{7}$ El EUSTAT no incorpora un desglose más detallado de los sectores principales (a excepción de la hostelería dentro del sector servicios) según el origen de las personas extranjeras ocupadas.
} 
hombres, a diferencia del resto, se distribuyen de forma bastante equilibrada entre los diferentes sectores económicos

\section{DISCUSIÓN}

Los resultados reflejan la progresiva presencia de la población extranjera en las zonas rurales vascas a lo largo de los últimos años; una progresión oscilante y discontinua que aparece marcada por la crisis y la recuperación económica. Los datos analizados reflejan con nitidez cómo su llegada inicial coincide con los años de expansión de la economía reduciéndose estos flujos como consecuencia de la crisis económica. Es interesante observar cómo hasta ese momento los ritmos de llegada a las zonas rurales y urbanas son similares asumiendo que las oportunidades de empleo en estas zonas son también elevadas. No obstante, esta dinámica no se mantiene en el periodo post-crisis, donde a partir de 2017 las zonas rurales recuperan de nuevo población extranjera, aunque más tardíamente que las zonas urbanas. Es decir, las recuperaciones poblacionales de los efectos de la crisis se detectan en las poblaciones rurales más tarde.

En este proceso, los efectos de la crisis sobre la modificación del perfil de la población extranjera se perciben en varias direcciones y, a pesar de que no aparecen cambios muy significativos, es interesante mencionar algunos de ellos. Por un lado, se observa un incremento de la población magrebí en el periodo post-crisis en detrimento de la población de origen europeo; una situación estrechamente ligada al ámbito laboral donde se detecta una progresiva demanda de mano de obra en la actividad agraria (y agroindustrial). Por el contrario, la población europea ha ido reduciendo su peso relativo motivado por la llegada de otros perfiles y como consecuencia, entre otros factores, de la reducción del empleo en actividades donde su presencia era relevante como la construcción. Es precisamente el origen uno de los rasgos que marcan la diferencia entre la población extranjera de las poblaciones rurales y las urbanas; en estas últimas, se detecta una sobre-representación de la población latinoamericana en detrimento de la europea y africana cuya presencia en las zonas rurales es más elevada. Una cuestión de interés y sobre la que resulta interesante seguir indagando más allá de la motivación laboral.

En relación al mercado de trabajo, el contraste entre la población extranjera y la total dibuja dos escenarios laborales que discurren en paralelo y apenas se conectan extrayendo resultados de interés. Junto a una presencia más reducida de las mujeres extranjeras en la actividad, es significativa la elevada tasa de paro que soporta el conjunto de la población extranjera, afectando nuevamente y, en mayor medida a las mujeres. Este desequilibro se traslada también a las actividades económicas de la población ocupada. En efecto, el análisis de la ocupación refleja actividades laborales asimétricas entre ambos colectivos, mucho más precarias y volátiles para la población extranjera. Los hombres se orientan hacia sectores refugio como la actividad agraria y construcción, y las mujeres se emplean en el servicio doméstico y la hostelería. Precisamente, la elevada proporción de mujeres de extranjeras en el ámbito doméstico refleja la vulnerabilidad del empleo femenino y cuestiona el alcance real de este tipo de empleo teniendo en cuenta el peso de la economía sumergida dentro de esta actividad.

Se trata de unos rasgos que, sin duda, contrastan con los de la población total que presentan, a priori, condiciones laborales y empleo mucho más estables. En el caso de los hombres destaca el peso del trabajo industrial, y entre las mujeres se observa una elevada 
representatividad de los sectores educativos, sanitarios o de comercio. Unos datos que reflejan la singularidad de la ruralidad vasca cuyo empleo en la actividad agraria, aunque cada vez más reducido recae sobre todo en los trabajadores extranjeros (fundamentalmente magrebíes) y en la que destaca la clara terciarización de su economía.

\section{CONCLUSIONES}

Esta investigación analiza la presencia de la población extranjera en las zonas rurales vascas como una oportunidad desde una óptica de sostenibilidad social y laboral, a partir de los principales rasgos sociolaborales y demográficos que presenta este colectivo. Se trata un colectivo en edad de trabajar, equilibrado desde la perspectiva de género, y que presenta una tasa significativa de la generación soporte. Elementos todos ellos muy favorables y que de manera conjunta pueden, en parte, contribuir al rejuvenecimiento y sostenimiento de las zonas rurales más desfavorecidas demográficamente. Si bien es cierto que la ruralidad vasca presenta rasgos en determinadas áreas y poblaciones que lo alejan de un tipo de ruralidad más deprimida, la motivación laboral y la búsqueda de empleo con mayor demanda ha favorecido que este colectivo se instale sobre todo en el caso de Álava en las poblaciones más pequeñas y con elevadas tasas de envejecimiento y masculinización.

No obstante, a pesar de este potencial, su efecto puede verse mitigado por factores que debilitan o incluso ponen en peligro el proceso migratorio a largo plazo. Por un lado, a pesar de la elevada representación de las mujeres extranjeras, su situación en el mercado de trabajo es mucho más débil. Aquellas que están ocupadas (un porcentaje reducido atendiendo en exclusiva a las cifras oficiales) trabajan prioritariamente en sectores poco cualificados e inestables haciéndose extensible esta situación a las mujeres que trabajan en la economía sumergida cuya dimensión se desconoce. Una situación que las convierte en colectivos mucho más vulnerables y con menor posibilidad de permanencia en función de las oportunidades laborales. Hay que tener en cuenta además la elevada proporción de personas que llegan solas, tanto hombres (sobre todo de origen africano) como mujeres (de origen latinoamericano fundamentalmente) por lo que su presencia dependerá en gran medida de las condiciones laborales que encuentren en las poblaciones rurales.

Para finalizar, la hipótesis de que la partía este trabajo relativa a la motivación laboral como factor de asentamiento de la población extranjera es validada, y se refleja con nitidez no solo en la presencia fluctuante de este colectivo en función del ciclo económico (y, por lo tanto, de las oportunidades laborales), sino también en el perfil demográfico que presenta la gran mayoría. Así mismo, su distribución territorial cercana a las poblaciones con mayor demanda de mano de obra avala esta idea. Aun así, conviene incorporar ciertos matices que pueden ayudar a comprender el proyecto migratorio a más largo plazo. Por un lado, el análisis indica que la salida de la población extranjera de las zonas rurales después de la crisis ha sido tardía y que, de alguna forma, este colectivo ha "resistido" durante más tiempo en las poblaciones rurales, constituyendo un aspecto muy significativo sobre el que conviene seguir indagando. Además, el periodo de recuperación económica ha favorecido que la población extranjera vuelva a asentarse en las zonas rurales a un ritmo similar previo a la crisis. Una recuperación que, sin embargo, desde una perspectiva temporal parece no haber influido sobre las ocupaciones laborales de los trabajadores extranjeros; unas actividades cuyos nichos de empleo permanecen en gran medida inalterables o incluso se deterioran en los últimos años dirigiéndose hacia ocupaciones más volátiles e inestables como los cuidados o la agricultura. Unos 
resultados que, a priori, podría estar reflejando un cierto estancamiento laboral de la población extranjera en las zonas rurales más allá del ciclo económico.

En definitiva, a pesar de la paulatina conversión en los últimos años de las zonas rurales en espacios generadores de empleo, los trabajadores extranjeros ocupan en gran medida actividades que la población autóctona ha ido abandonando (agricultura) o reemplazando (cuidados) junto a otras que han ido apareciendo según el ciclo económico lo que las convierte en nuevos residentes mucho más volátiles. Una situación que exige repensar el papel de la población extranjera en las zonas rurales más a largo plazo, y que obliga a planificar políticas sociales y de vivienda que hagan más sostenible su presencia en las zonas rurales abocada en este momento al devenir de la economía, y del empleo

\section{ORIENTACIONES FUTURAS}

El análisis estadístico que se ha empleado en este trabajo necesita del apoyo de una perspectiva cualitativa que permita conocer las trayectorias laborales y las perspectivas de futuro de las personas extranjeras en las zonas rurales. Para ello, junto al testimonio directo de este colectivo, es imprescindible conocer también la percepción de informantes clave vinculados a los ámbitos laboral y social que aporten una visión mucho más amplia. Descubrir los factores sociodemográficos y/ o laborales que influyen en la permanencia en las áreas rurales de las personas extranjeras son herramientas imprescindibles para los poderes públicos a la hora de diseñar y abordar políticas de empleo y social que favorezcan el mantenimiento e incremento de este colectivo. Un objetivo de estudio de gran interés y relevancia sobre el que es necesario seguir indagando.

\section{AGRADECIMIENTOS}

Las investigadoras quieren agradecer a las técnicas del Instituto Vasco de Estadística (EUSTAT) por su interés y apoyo prestado en la búsqueda y elaboración de datos estadísticos que han sido necesarios para esta investigación.

\section{REFERENCIAS}

Ainz, Ma . J. (2001). El caserío vasco en el país de las industrias. Serie Estudios, 147. Ministerio de Ministerio de Agricultura, Pesca y alimentación, Secretaría General Técnica.

Alderdi, J. C. (2016). Límites en la promoción rural en el País Vasco tras dos décadas de programas de desarrollo rural. Investigaciones geográficas, (66), 59-78. https://doi.org/10.14198/ingeo2016.66.04

Camarero, L. (2009). La población rural de España. De los desequilibrios a la sostenibilidad social. Colección Estudios, 27. Fundación La Caixa. Recuperado de: https://fundacionlacaixa.org/documents/10280/240906/vol27_es.pdf (04/10/2021). 
Camarero, L., y Oliva, J. (1999). La ruralidad en la sociedad itinerante: breve panorámica de la ruralidad vasca. Inguruak. Revista Vasca de Sociología y Ciencia Política, (25), $125-138$.

Camarero, L., y Sampedro, R. (2019). Despoblación y ruralidad transnacional: crisis y arraigo rural en Castilla y León. Economía Agraria y Recursos Naturales, 1(19), 5982. https://doi.org/10.7201/earn.2019.01.04

Camarero, L., Sampedro, R., y Oliva, J. (2013). Trayectorias ocupacionales y residenciales de los inmigrantes extranjeros en las áreas rurales españolas. Sociología del Trabajo, (77), 69-91. Recuperado de: https://revistas.ucm.es/index.php/STRA/article/view/60574 (04/10/2021).

Collantes, F., Pinilla, V., Sáez, L. A., y Silvestre, J. (2014). Reducing depopulation in rural Spain: the impact of immigration. Population, Space and Place, 20(7), 606-621. https://doi.org/10.1002/psp.1797.

Danson, M., y Jentsch, B. (2012). International migration and economic participation in small towns and rural areas-cross-national evidence. Migration Letters, 9(3), 215-224. https://doi.org 10.33182/ml.v9i3.93,

Escribano, J. (2012). El valor de los servicios educativos y sanitarios en los procesos de atracción y mantenimiento de población en medio rural. AGER, Revista de Estudios sobre Despoblación y Desarrollo Rural, (13), 11-51. https://doi.org/10.4422/ager.2011.07.

Gadea, M. E., De Castro, C., Pedreño, A., y Moraes, N. (2015). Jornaleros inmigrantes en la agricultura murciana: reflexiones sobre crisis, inmigración y empleo agrícola. Migraciones, (37), 149-169.

Gadea, M. E., De Castro, C., Pedreño, A., y Ramírez, A. J. (2016). Almaceneras: Género y trabajo en los almacenes de manipulación de fruta en la región de Murcia. Revista Andaluza de Antropología, (11), 121-145.

Gualda, E. (2012). Migración circular en tiempos de crisis. Mujeres de Europa del Este y africanas en la agricultura de Huelva. Papers Revista de Sociología, 97(3), 613-640. https://doi.org/10.5565/rev/papers/v97n3.436

Murua, J. R., y Múgica, B. (2016). Inmigración y emprendimiento rural: ejes de innovación de las políticas de desarrollo rural. ITEA, 112(4), 438-457. Recuperado de: https://itea.org/aidaitea/files/itea/revistas/2016/1124/438457\%20ITEA\%20112-4.pdf $(04 / 10 / 2021)$.

Ray, J. F., y Scott, M. H. (2020). The new geography of rural migration: EU11 migrants in rural Norway. Journal of Rural Studies, (75), 125-131. https://doi.org/10.1016/j.jrurstud.2020.01.014

Rivera, M. J. (2009). La neorruralidad y sus significados. El caso de Navarra. Revista Internacional de Sociología, 67(2), 413-433. https://doi.org/10.3989/RIS.2008.05.11

Sampedro, R. y Camarero, L. (2016). Inmigrantes, estrategias familiares y arraigo: las lecciones de la crisis en las áreas rurales. Migraciones (40), 3-31. https://doi.org/10.14422/mig.i40y2016.008

Sánchez-Flores, S., Royo, I., Lacoma, J., Marí, E., y Benlloch, C. (2014). Mujeres inmigrantes emprendedoras en el medio rural. Factor para la sostenibilidad económica y social de las áreas rurales de la Comunidad Valenciana. AGER, Revista de Estudios 
sobre Despoblación y Desarrollo Rural, (16), 69-109. https://doi.org/10.4422/ager.2013.05.

Solé, A., Guirado, C. y Solana. M. A. (2012). Cambios en la dinámica demográfica y migratoria del Pirineo catalán. Análisis sociolaboral de la población extranjera., (12), 51-90. https://doi.org/10.4422/ager.2011.02

Soronellas, M., Bodoque, Y., Blay, J., Roquer S., y Torrens, R. (2014). Inmigrar a la Cataluña rural. Contextos de ruralidad y migraciones de mujeres extranjeras hacia pequeños municipios. AGER, Revista de Estudios sobre Despoblación y Desarrollo Rural, (16), 111-148. https://doi.org/ 10.4422/ager.2014.01

Torres, F., y Gadea, E. (2010). Inserción laboral de los inmigrantes, estructura etnofragmentada y crisis económica. El caso del Campo de Cartagena (Murcia). Sociología del Trabajo, (69), 73-94.

Urrestarazu, E., y Galdos, R. (2019). Hacia una asimilación socioeconómica del campo y la ciudad: cambios recientes y diversidad en las áreas rurales del País Vasco. Lurralde, (42), 131-150.Recuperado de: https://ingeba.org/lurralde/index.htm (04/10/2021).

Vercher, N., Escribano, J., y Valero, D. E. (2019). Models de vida femenins en el medi rural. Un cas d'estudi a la Sierra del Segura (Albacete). Documents d'Anàlisi Geogràfica, 65(1), 139-161. Recuperado de: https://raco.cat/index.php/DocumentsAnalisi/article/view/v65-n1-vercher-escribanovalero $(04 / 10 / 2021)$.

Vercher, N., Escribano, J., y Valero, D. E. (2020). Redes de apoyo al emprendimiento femenino en territorios rurales. OBETS. Revista de Ciencias Sociales, 15(1), 317-356. https://doi.org/10.14198/OBETS2020.15.1.11. 


\section{EXTENDED ABSTRACT ${ }^{8}$}

The aim of this paper is to present the main results of a quantitative study focused on the sociodemographic profile and labour market situation of the foreign-born population that has settled in Basque rural areas over the last two decades. The increase of migrant residents and their role in rural municipalities is an interesting topic due to the socioeconomic impact that the arrival of this group has on these areas, and in addition it is a topic that is somewhat unexplored in the Basque context.

This research also reflects the huge potential of this group for slowing the process of depopulation and revitalizing rural areas, especially in those municipalities most affected by depopulation: recent statistics examined show a group whose average age is under 35 and gender balanced. Besides this, one main characteristic is heterogeneity regarding the origin of this population, the most significant groups proceeding from Morocco and South America.

Thus, Basque rural areas provide a very interesting scenario for studying the sociological dynamics connected to the migrant population and its social and economic integration into the territory. Concerning the period studied, several changes have taken place, not only in the social field but also at the economic and labour level, contributing in certain respects to the transformation and diversity of Basque rural areas.

However, the results concerning the position of migrants in the labour market are probably one of the main contributions of this study, providing a fundamental perspective for understanding the social and economic impact of this group on rural areas. Different studies demonstrate how over the last twenty years these areas have been generating employment opportunities not only in the agrarian sector but also (and mainly) in the service sector. The research therefore shows that labour motivation proves to be the main reason for migrants installing themselves in these places, although this is strongly dependent on the economic cycle, with the crisis of 2008 and subsequent years having significant effects, which are also studied in this research.

Different hypotheses are posited: the first hypothesis is that labour motivation is the main factor triggering the migration project; a second hypothesis is connected to the sociodemographic profile of migrants, with the purpose of knowing whether this varies between rural and urban areas; a third hypothesis shows how market segmentation is connected to the migrant workers' place of birth.

The investigation uses a quantitative methodological approach based on secondary data provided by the Basque Statistical authorities and obtained ad hoc for this study; concretely, the principal population and labour statistics were examined. On the one hand, to construct the demographic characterization, continuous Population Censuses for the years 2019, 2016, 2011, 2006 and 2001 were analyzed, as well other data proceeding from the Basque Statistical Institute. It is relevant to consider the entire period in order to obtain a broader perspective on migration at different stages: the years prior to the massive influx of migrants (2001), the period before the economic crisis (2006), the effects of that crisis (2011), the decline in migration to rural areas (2016) and, finally, the most recent data available, with the aim of obtaining the most up-to-date scenario.

Regarding their representation in the labour market, population, not municipality, was taken into account as a unit of analysis, to try to avoid statistical overrepresentation. All

\footnotetext{
${ }^{8}$ Traducción exclusiva de los autores / Authors' exclusive translation.
} 
the data were examined on the basis of three main variables: gender, age and country or continent of birth. In some cases, it was considered interesting for the purpose of the study to establish a comparison between the foreign-born population and the local population.

The results show how labour motivation is the main factor for arriving in rural areas, confirming the first hypothesis, while the data indicates that, compared to other studies, departure from rural areas following the crisis was delayed and the migrant population "resisted" for longer in rural territories, probably due to lower prices. The data clearly reflect the progressive increase of the migrant population in Basque rural areas over the last two decades; however that evolution has oscillated, showing a certain discontinuity, and has been marked by the crisis and economic recovery. In effect, the weight of the economic situation and, particularly, the increase of labour opportunities becomes clear. The data explored accurately reflect how the arrival of the migrant population ties in with the years of a very positive economic dynamic in Spain, with a reduction of these population flows resulting from the economic crisis after the year 2008. However, it is very interesting to observe how prior to that situation the data on arrival in rural and urban areas develop in parallel and are very similar. This dynamic, however, is not maintained in the post-crisis period: following the year 2017 rural areas again recover their foreignborn population although later than urban areas.

Regarding the second and third hypotheses, in connection with the labour market and the comparative situation of the foreign-born and the local populations, the results highlight two very dissimilar labour scenarios that flow in parallel, with some of the reflections obtained proving to be very interesting. Together with migrant women having a less representative role in the legal labour market, the high unemployment rate amongst migrants, which affects women to a greater extent, is very relevant. This imbalance can be observed again within the working population. Thus, the analysis shows that the labour conditions are much more asymmetric and volatile. In the case of foreign-born men, their jobs are inserted in precarious sectors such as agrarian activity as well as construction. Regarding women, the percentage of those working in the care sector, tourism and the catering sector is very significant. Indeed, the extraordinary rate of women employed in the care sector reflects the vulnerability of women's jobs and - of greater relevance questions the real dimension of these kinds of jobs, taking into account the shadow economy within this particular activity. This situation is especially relevant in the case of elderly care, a form of work that is mainly developed by South American women. Many of these women have no work or residence permits, and it is easier to avoid legal control in rural localities.

This information contrasts with the job activities of local people that present, a priori, much more stable labour conditions: a high rate of men work in industry, presenting much better working conditions, while women have a high representation in education, the health sector and the public administration. These data reflect the singularity of Basque rural areas, showing that although employment in the agrarian sector is becoming increasingly reduced, it is the migrant population (mainly people from the Maghreb) that is working in that sector, mostly oriented to basic activities in wine production.

Finally, the study calls for the development of a qualitative analysis, combined with the quantitative ones, in order to know the work histories and narratives of foreign-born people in rural areas. This would undoubtedly contribute a complementary perspective to the research that has been realized. 


\section{CONTRIBUTION POR AUTORES}

\begin{tabular}{|c|l|c|c|c|}
\hline \multicolumn{1}{|c|}{ ITEM } & Beatriz Izquierdo & Julia Shershneva & Patricia Campelo \\
\hline 1 & Conceptualización & $90 \%$ & $5 \%$ & $5 \%$ \\
\hline 2 & Tratamiento de datos & $70 \%$ & $25 \%$ & $5 \%$ \\
\hline 3 & Análisis formal & $50 \%$ & $25 \%$ & $25 \%$ \\
\hline 4 & Acceso financiación & $0 \%$ & $0 \%$ & $0 \%$ \\
\hline 5 & Investigación & $70 \%$ & $20 \%$ & $10 \%$ \\
\hline 6 & Metodología & $70 \%$ & $20 \%$ & $10 \%$ \\
\hline 7 & Gestión del proyecto & $70 \%$ & $25 \%$ & $5 \%$ \\
\hline 8 & Recursos & $70 \%$ & $0 \%$ & $0 \%$ \\
\hline 9 & Software & $0 \%$ & $0 \%$ & $0 \%$ \\
\hline 10 & Supervisión & $0 \%$ & $25 \%$ & $5 \%$ \\
\hline 11 & Validación & $70 \%$ & $10 \%$ & $0 \%$ \\
\hline 12 & Visualización & $90 \%$ & $20 \%$ & $0 \%$ \\
\hline 13 & Redacción (borrador) & $80 \%$ & $10 \%$ \\
\hline 14 & $\begin{array}{l}\text { Redacción final (revisión } \\
\text { y edición) }\end{array}$ & $70 \%$ & $20 \%$ & \\
\hline
\end{tabular}

Para más información, visitar CRediT: https://casrai.org/credit/ 